\title{
ACCIONES Y FUNDAMENTOS DEL TRABAJO DEL CUERPO MÉDICO ESCOLAR EN EL URUGUAY (1907-1927). LA CONSTRUCCIÓN DE LA NORMALIDAD EN LOS CUERPOS EN EL MAGISTERIO
}

\author{
Actions and Foundations of the Work of the School Medical Corps in Uruguay (1907-1927). \\ The Construction of Normality in the Bodies of the Teaching Community
}

\author{
Fernanda Sosa ${ }^{1}$ \\ ORCID: 0000-0002-5184-0582 \\ ${ }^{1}$ Consejo de Formación en Educación, Administración Nacional de Educación Pública, Uruguay. \\ Correo: totifernanda@gmail.com
}

Recibido: 23/04/2020

Aceptado: $31 / 10 / 2020$

Resumen: En este trabajo se analiza el funcionamiento del Cuerpo Médico Escolar (CME) en el Uruguay entre 1907 y 1927 y se sostiene que uno de los ámbitos de mayor influencia de sus acciones lo constituye la construcción de normalidad en los cuerpos de maestras, maestros y estudiantes magisteriales. La normalización es un concepto foucaultiano que refiere a la regulación de los cuerpos de los individuos (disciplinas) y de las poblaciones (biopolíticas) y que orientará el análisis de las acciones del CME, particularmente las desarrolladas con estudiantes magisteriales, concursantes para cargos docentes, y en el reconocimiento anual del estado sanitario del magisterio. Las acciones llevadas adelante por el CME se fundan en argumentos que provienen del higienismo, superpuestos con argumentos propios de la eugenesia. Para este trabajo se analizaron reglamentaciones, sesiones e informes internos del Cuerpo Médico Escolar; artículos en Anales de Instrucción Primaria; Informes del CME en las Memorias de Instrucción Primaria y manuales de Anatomía, Fisiología e Higiene y de Economía Doméstica.

Palabras clave: Cuerpo Médico Escolar; normalización; magisterio; higienismo; eugenesia.

\begin{abstract}
In this work, the functioning of the School Medical Corps (CME, for its Spanish acronym) in Uruguay between 1907 and 1927 is analyzed and it is argued that one of the most influential areas of its actions is the construction of normality in the teaching corps of teachers and student teachers. Normalization is a Foucaultian concept that refers to the regulation of the bodies of individuals (disciplines) and populations (biopolitics) and will guide the analysis of the actions of the CME, particularly those developed with student teachers, contestants for teaching positions, and in the annual recognition of the health status of teachers. The actions carried out by the CME are based on arguments that come from hygienism, superimposed with arguments typical of eugenics. For this work, an analysis was made of regulations, sessions and internal reports of the School Medical Corps; articles in Annals of Primary Instruction; CME reports in Primary Instruction Reports and manuals on Anatomy, Physiology and Hygiene and Home Economics.
\end{abstract}

Keywords: School Medical Corps; normalization; teaching; hygienism; eugenics. 


\section{Introducción}

El presente trabajo ubica la creación, acción y fundamentos del Cuerpo Médico Escolar (CME) en el Uruguay y hace foco con posterioridad en las ideas y las prácticas que, desde el CME, contribuyeron a construir patrones de normalidad en el magisterio. El período a estudio comienza en 1907 con el análisis de documentos que proponían la creación de un Cuerpo Médico Escolar, Cuerpo que se crea en el año 1908, y culmina en 1927, año en que se modifica el Reglamento del Cuerpo Médico Escolar y su institucionalidad cambia debido a la creación de la figura de las visitadoras escolares ${ }^{1} \mathrm{y}$ a una nueva estructura de funcionamiento.

El CME funcionó en momentos en que numerosas enfermedades contagiosas azotaban a la población; de ahí que propusieran una serie de medidas que atendían a la minimización de los contagios: distancia social en el aula, vacunaciones, atención a los preceptos de higiene en las escuelas del país, etc. Para el alumnado que se contagiaba de alguna enfermedad se proponía el confinamiento en el hogar por una cantidad de días acorde a las duraciones de los períodos de contagio de cada enfermedad. Pero la acción fue más allá de la profilaxis, detección y precaución ante las enfermedades contagiosas, porque el CME se ocupó también de la promoción de clases diferenciales y escuelas especiales para "retardados mentales" y para "sordo-mudos". Uno de los ámbitos de mayor incidencia lo constituye la selección que el CME realizaba de estudiantes magisteriales y de concursantes por puestos en el magisterio, junto con la cesación temporal o definitiva de maestros/as de acuerdo con el resultado en el reconocimiento sanitario anual del magisterio, como se verá.

Michel Foucault aporta nociones que permiten interpretar los desarrollos planteados en este artículo. Foucault (1975/1996) llama disciplinas a los "métodos que permiten el control minucioso de las operaciones del cuerpo, que garantizan la sujeción constante de sus fuerzas y les imponen una relación de docilidad-utilidad" (p. 141). Las técnicas disciplinarias fueron surgiendo en distintos ámbitos, como procesos menores, con orígenes diferenciados, de localización diseminada, que fueron imponiéndose para responder a determinadas exigencias coyunturales y luego se repiten, se imitan, se apoyan unos sobre otros hasta constituirse en un

\footnotetext{
${ }^{1}$ Con el cambio de reglamentación, las visitadoras escolares pasaron a ocuparse de la medición de talla, peso, vigilancia de agudeza visual y auditiva y del estado higiénico en general. Complementaban al personal médico en las visitas higiénicas a hogares, visitaban a domicilio a estudiantes que faltaran para tener información de posibles contagios, entre otras tareas (Reglamento del Cuerpo Médico Escolar, 1927).
} 
método general (Foucault, 1975/1996). Estos cambios se dieron en la modernidad, a la que Foucault identifica como sociedad disciplinaria. A esta idea de disciplina que opera en los cuerpos, le agrega otra idea de distinto nivel: la de biopolíticas. Las biopolíticas (Foucault, 2000 , p. 220) constituyen una tecnología de poder que no se dirige al cuerpo, sino a la población. Los objetos de saber y de control de las biopolíticas son, entonces, los procesos que involucran a las poblaciones ${ }^{2}$.

Como explica Edgardo Castro (2004) —en la entrada correspondiente a norma en su diccionario sobre Foucault - el concepto de normalización se refiere al proceso de regulación de la vida de los individuos (disciplinas) y de las poblaciones (biopolíticas). Las sociedades modernas son sociedades de normalización, explica Castro; no alcanza con la idea de sociedades disciplinarias expresada en Vigilar y Castigar (Foucault, 1975/1996), sino que hay que ampliar a la idea de sociedades de normalización a partir de planteos foucaultianos posteriores.

El objeto de este artículo comprende además la fundamentación de las acciones emprendidas por el CME. Estas se basaban en argumentos higienistas que convergían con argumentos eugenésicos.

El artículo está dividido en dos grandes partes. En la primera se analiza la creación, el funcionamiento y el alcance de las acciones desplegadas por el CME revisando sus fundamentos y la manera en que opera la normalización. En la segunda parte se hace foco en la normalización en el magisterio revisando el currículo, el examen de selección de estudiantes magisteriales y de concursantes como maestros nacionales y el reconocimiento anual del estado sanitario del magisterio.

\section{La creación y el funcionamiento del Cuerpo Médico Escolar}

El Uruguay de principios del siglo XX transitaba, junto con la modernización económica, social y política, un nuevo orden de sentimientos que el historiador José Pedro Barrán (2011) ha caracterizado como la etapa del "disciplinamiento"; coexistía una "nueva sensibilidad civilizada" con algunos rasgos de la anterior "barbarie" (p. 215). Un elemento clave en esta nueva sensibilidad fue la preocupación por la conservación de la salud del cuerpo. Para prevenir la

\footnotetext{
${ }^{2}$ En Defender la sociedad (Foucault, 2000) se ubica la aparición de las disciplinas en los siglos XVII-XVIII (p. 219) mientras que las biopolíticas, nueva tecnología de poder que no excluye a la primera, aparece en la segunda mitad del siglo XVIII (p. 219).
} 
enfermedad individual y las "enfermedades sociales", la ciencia aportaba conocimientos a través de una disciplina denominada Higiene. El movimiento higienista estuvo formado por "un grupo de médicos y sociólogos que veían en los programas de salud pública una forma de mejorar el perfil sanitario urbano" (Lavrin, 2005, p. 132).

El culto a la salud condujo a la medicalización de la sociedad en el marco de la finalización del proceso de secularización de la sociedad uruguaya. El poder médico (Barrán, 1992, p. 13) emana de su saber, de la ciencia, un saber requerido para el mantenimiento de la salud. El vigor, la fuerza, la "riqueza" del cuerpo, eran los bienes por obtener, y su debilidad, su “pobreza”, el mal a exorcizar (Barrán, 2011, p. 249).

Pero la protección de la propia salud requería también de la protección de la salud ajena. Las epidemias, las enfermedades venéreas — particularmente la sífilis-, la tuberculosis y el alcoholismo eran vistos como males sociales que había que atender. Paulina Luisi ${ }^{3}$ (1919, p. 11) — una de las médicas del Cuerpo Médico Escolar- sostenía que la sífilis, la tuberculosis y el alcoholismo eran el "triunvirato destructor de nuestra especie" contra el que había que desplegar una amplia acción educativa.

Como se verá, el discurso pedagógico incorporó conceptos provenientes del discurso médico (higienista y eugenista) en el currículo y en múltiples aspectos de la vida de las escuelas de principios del siglo XX. Silvana Darré (2005, p. 201) explica que "la especificidad del discurso pedagógico radica en su posibilidad de vehiculizar otros discursos sociales, políticos, jurídicos, médicos", ya que reúne lo que es seleccionado del conjunto de otros discursos para su circulación en términos pedagógicos.

La incorporación de la Higiene al currículo escolar uruguayo data de fines del siglo XIX ${ }^{4}$ pero a principios del siglo XX las conferencias, trabajos presentados a congresos y artículos publicados en Anales de Instrucción Primaria son múltiples y anticipan la mayor incidencia del discurso higienista en los centros educativos, generando un ambiente propicio para la creación del CME.

\footnotetext{
${ }^{3}$ Para una biografía de Paulina Luisi, ver Sapriza (1988).

${ }^{4}$ La Fisiología e Higiene se había incorporado al currículo en 1877 (Decreto-Ley de Educación Común) y la influyente obra de 1874 de José Pedro Varela denominada La educación del pueblo fundamenta la necesaria incorporación al currículo de esa asignatura para la preservación de la salud (Varela, 1964, p. 146)
} 


\section{Creación del Cuerpo Médico Escolar: antecedentes y fundamentos}

Sebastián B. Rodríguez (1907) presentó un trabajo al tercer Congreso Médico Latinoamericano que consistió en un Anteproyecto para la creación del Cuerpo Médico Escolar. En este anteproyecto, claramente enmarcado en el higienismo, figura una extensa lista de cometidos, la inspección higiénica de las escuelas, la vacunación y revacunación obligatoria para toda persona en un centro educativo y una extensa normativa para la profilaxis de las enfermedades en las escuelas.

Vale la pena detenerse en los fundamentos presentados por Rodríguez para este anteproyecto. La intención era intervenir en lo edilicio, la limpieza, la comida y además en lo pedagógico, fundando formas de trabajo cotidiano que atendieran a la higiene de los cuerpos tal como la entendía la medicina de entonces. Una primera cuestión que plantea el anteproyecto es la defensa de la profilaxis. La medicina ya no se trataba de curar a los enfermos, sino que se adentraba en evitar las enfermedades por medio de la difusión de la Higiene. Sostiene que "el grupo escolar” es el que debía merecer más atención por parte de los poderes públicos y las autoridades médicas respectivas (Rodríguez, 1907, p. 24). Describe en su anteproyecto las escuelas de veinticinco o treinta años antes (fines del siglo XIX), con falta de luz, de ventilación, de aseo, con hacinamiento, comida insuficiente y desagradable y baños sucios. Agrega como elementos perniciosos en esas antiguas escuelas al silencio absoluto que se imponía en las clases, la memorización de las lecciones y la "práctica pura del cerebro, anemia total del cuerpo" (Rodríguez, 1907, p. 26). La incidencia de lo médico en lo pedagógico se evidencia en estas propuestas.

Rodríguez recorre los trabajos en materia de higiene escolar realizados en varios países latinoamericanos, algunos de ellos ya habían creado Cuerpos Médicos Escolares (Argentina, México, Costa Rica) mientras otros, que aún no los tenían, prestaban asimismo atención a cuestiones vinculadas con la higiene escolar poniendo en práctica otros mecanismos (Brasil, Chile, Paraguay). Su convicción lo lleva a proponer frente a colegas de catorce nacionalidades presentes en el mencionado congreso que: "El 3er. Congreso Médico Latino-Americano declare que es de urgente necesidad la creación y organización definitiva del Cuerpo Médico Escolar en todos los países de América” (Rodríguez, 1907, p. 40). 
Tres meses después, Abel J. Pérez (1907), inspector nacional de Instrucción Primaria (cargo de máxima jerarquía en el sistema de educación pública primaria), pronunció una Conferencia en el Ateneo del Uruguay denominada “Apuntes sobre higiene escolar". En esta conferencia abogó por la creación de un Cuerpo Médico Escolar — como ya lo había propuesto en la Memoria de Instrucción Primaria de 1904-1905 logrando la asignación presupuestal para la creación del Cuerpo. El primer argumento que brinda para esta creación es que, al seguir "los consejos de una ciencia profunda, de una experimentación constante y de una abnegación inagotable" (Pérez, 1907, p. 259) se había logrado que decreciera la morbilidad infantil. Propone pensar en la higiene escolar (locales, menaje, materiales) y en los mismos escolares en su conjunto o aisladamente y también como posibles vehículos de contagio (Pérez, 1907, p. 260).

Pérez brinda en su conferencia extensas consideraciones acerca de los cambios que en nombre de la higiene deben realizarse en locales escolares; en su menaje: bancos que respeten a la columna vertebral, incluso propone avanzar hacia la mesa-banco higiénica individual, en lugar de la de dos plazas, para "aislar higiénicamente al alumno" (Pérez, 1907, p. 262); en los materiales de estudio: discute el tamaño, color, inclinación de las impresiones, los mapas, etc.

Pero las ideas de Pérez van más allá de los espacios, muebles y materiales de estudio que respeten las pautas higiénicas. Acerca de los estudiantes propone una estricta vigilancia para detectar enfermedades infecto-contagiosas en ciernes y para detectar al "alumno que sin estar ostensiblemente enfermo, lleva en sí el germen de todas las dolencias, en su organismo símbolo de todas las miserias orgánicas y fisiológicas.” (Pérez, 1907, p. 264). La línea de demarcación entre sanos y enfermos se corre. Junto a la enfermedad, se coloca a la "enfermedad en ciernes" y aparece la figura del niño débil, que inaugurará otros ámbitos de intervención.

Para Pérez estos niños que "llevan el germen de todas las dolencias" son producto de varias miserias combinadas que producen un "ser incoloro, anémico, sin energías, campo fértil de todas las dolencias físicas y de todas las subversiones morales" (Pérez, 1907, p. 268). A estas expresiones se agregan otras que portan una connotación de clase social: la vida en los conventillos, la alimentación escasa, el alcoholismo, son los males que generan descendencia en condición de debilidad según Pérez. Para enfrentar estas dolencias que van degenerando la raza (Pérez, 1907, p. 269) propone: alimento abundante y genuino, ejercicios físicos científicos, colonias infantiles a las orillas del mar, en las sierras o en el interior del país, ropas abrigadas e higiénicas, medicación en caso de enfermedad, todo esto "puede transformar estos niños, 
combatir sus dolencias, hacerlos sanos, robustos; elementos de acción para lo futuro, ciudadanos en fin de una democracia activa, y no elementos prematuramente cansados, seres fracasados, antes de empezar a vivir y luchar" (Pérez, 1907, p. 269)5.

La propuesta de mejoramiento del medio como manera de incidir en el mejoramiento de la raza proviene de las denominadas eugenesias ambientalistas. Se requerirían mayores indagaciones y análisis para ubicar el pensamiento del inspector Pérez, pero corresponde señalar que la idea de "degeneración de la raza" proviene del pensamiento eugenésico. La eugenesia, definida por su fundador Francis Galton como la "ciencia que se ocupa de todas las influencias que mejoran las cualidades innatas de una raza" (Galton en Reggiani, 1914, p. 17), tuvo incidencia en ámbitos médicos montevideanos ${ }^{6}$. "Galton creía que las cualidades morales y las capacidades intelectuales — el talento, como las llamaba — se transmitían hereditariamente, de la misma forma que el color de ojos y el cabello" (Reggiani, 2019, p. 18). Tras el redescubrimiento de las teorías mendelianas que demostraban que los caracteres adquiridos no se transmitían, el campo de la eugenesia quedó dividido entre hereditaristas y ambientalistas (Reggiani, 2014, p. 3). Paulina Luisi, reconocida eugenista (Sapriza, 2008), sostenía: "La herencia puede, hasta cierto punto, ser modificada por una buena higiene - pero para que esta pueda ser útil, es imprescindible un medio apropiado- y es el escollo contra el que se estrellan las mejores voluntades" (Luisi, 1919b, p. 53).

Las eugenesias ambientalistas otorgaban un papel clave a la difusión de pautas higiénicas y, según Reggiani (2019), la preocupación por el mejoramiento de las condiciones medioambientales en que se desarrollaba la infancia le dio un perfil característico a la eugenesia latinoamericana.

Diego Armus (2016) señala que algunos estudios sobre eugenesia enfatizan en la existencia de una eugenesia positiva, medioambientalista y educativa frente a una eugenesia negativa. La primera, propia del mundo latino, dio un lugar marginal a la esterilización y a violentos métodos de selección. La segunda, propia del mundo anglosajón, promovió la esterilización, la castración e incluso la eutanasia de seres indeseables o que conciban hijos con

\footnotetext{
${ }^{5}$ Esta cita condensa los contenidos presentados por Lionetti (2011) acerca de la intervención del discurso médico en la escuela con la intención de actuar sobre el cuerpo de los escolares hacia la preservación de la salud en su condición de futuros ciudadanos de la república.

${ }^{6}$ Según Barrán (1995, p. 206) fue la creencia dominante entre los médicos del Novecientos.
} 
enfermedades hereditarias. Pero Paulina Luisi (1916) propuso medidas propias tanto de la llamada eugenesia positiva como de la negativa ${ }^{7}$.

Marisa Miranda y Gustavo Vallejo han revisado los conceptos de eugenesia positiva y negativa y proponen distinguir entre eugenesias de coercitividad explícita y eugenesias de coercitividad disimulada. Siguiendo estos planteos, el centro de la discusión se desplaza, ambas eugenesias construyen la idea de "otro". Mientras en la eugenesia anglosajona la exclusión del "otro" no tenía tapujos, en la eugenesia latina se daba una especie de inclusión por asimilación, el “otro" debía dejar de ser lo que era (Miranda y Vallejo en Armús, 2016, p. 155).

Ahora bien, Reggiani (1914) estima que los ideales de la eugenesia se confundían con nociones de salud física y bienestar corporal: "Es por ello que a veces no resulta fácil distinguir las medidas genéricamente higiénicas de las específicamente eugenésicas, es decir, de aquellas preocupadas por la herencia y el mejoramiento de las condiciones psicofísicas de las generaciones futuras” (pp. 1-2). En términos similares se expresa Dogliotti (2012, p. 117). También Espiga (2015, p. 125), quien afirma: “El discurso eugenésico estuvo implícito en las disertaciones y acciones higienistas".

La conferencia de Abel Pérez prosigue con una propuesta de cometidos para el Cuerpo Médico Escolar y con la propuesta de creación de una libreta sanitaria escolar. En esta libreta sanitaria se registrarían las observaciones realizadas a cada alumno (desarrollo físico, funcionamiento de órganos, afecciones sufridas y antecedentes sanitarios de la familia). No sólo se registrarían las afecciones sufridas, sino "las que puede sufrir, atendida su conformación, su aspecto y los signos visibles de las diversas manifestaciones de su organismo" (Pérez, 1907, p. 272). Para completar estas libretas, los médicos debían dedicar tiempo a su realización ya sea con niños “sanos, sospechosos o enfermos” (Pérez, 1907, p. 273).

\footnotetext{
${ }^{7}$ Barrán (1999) señala como un sello del eugenismo uruguayo al vínculo entre reforma social y eugenesia. Esto se condice con el análisis que realiza Stepan (1991) del Código del Niño de 1934 como la pieza paradigmática para entender la eugenesia no solo uruguaya sino latina en general. Esta autora plantea que la eugenesia latina incorporó medidas de protección social, a diferencia, por ejemplo, de los Estados Unidos, en donde la eugenesia excluyó la preocupación por la salud y el bienestar social. Para Barrán (1999) el eugenismo uruguayo se caracterizó por preferir el término especie en lugar de raza, siendo Paulina Luisi una excepción. Se priorizó la lucha contra la sífilis, el alcoholismo y la tuberculosis y la principal línea de acción fue el control de la procreación a través de la existencia de Consultorios Médicos Prenupciales para procurar que solo las personas sanas engendraran descendencia. Acudir a estos consultorios no constituyó una práctica obligatoria. Las ideas eugenésicas tuvieron además incidencia en la despenalización del aborto entre 1934 y 1938 (Sapriza, 2011) y en la fundamentación de dos leyes inmigratorias 1932 y 1936 - que contienen argumentos eugenésicos en sus discursos de justificación (Facal, 2002).
} 
La sospecha de enfermedad, que se establece a partir de la constatación de la debilidad aparente del alumno, también justifica intervenciones igual que en el caso del alumno enfermo. Esta propuesta se consolida cuando se crea el CME; en lugar de libretas sanitarias se denominan fichas sanitarias y serán analizadas en el siguiente apartado.

\section{Acciones del Cuerpo Médico Escolar (1907-1927)}

¿Cómo actúa la normalización? Por un lado, la disciplina normaliza ${ }^{8}$, esto es la normalización disciplinaria (Foucault, 2000, p. 75) que se produce a partir de la fijación de una norma que permite distinguir entre la gente, los gestos y los actos que se ajusten a ese modelo, que serán los normales, y los que no se ajusten, que serán los anormales.

Foucault plantea que, como se trata de cumplir la norma - la norma tiene un carácter prescriptivo-, la normalización disciplinaria podría denominarse normación. Por otro lado, la normalización en sentido estricto es aquella en la que lo normal se fija a partir de curvas de normalidad ${ }^{9}$. En las acciones del CME pueden verse ambos procesos de normalización. Se presentarán brevemente las acciones generales del CME en el período seleccionado para poder ubicar en contexto las tareas que el Cuerpo llevó adelante en el magisterio ${ }^{10}$.

En 1908 se crea el CME con el cometido de "asesorar al Ministerio de Industrias, Trabajo e Instrucción Pública y a la Dirección General de Instrucción Primaria, sobre todas las cuestiones que afecten a la higiene de la escuela, la higiene de los escolares y la higiene del personal docente" (Reglamentación para el ejercicio de sus funciones, 1909, p. 207). Dos años después se aprueba un Reglamento del Cuerpo Médico Escolar Nacional (1910).

Un repaso en ambos documentos de los asuntos en los que se requeriría asesoramiento al CME da una dimensión de su alta incidencia por la cantidad de temas implicados y lo definitivos

\footnotetext{
${ }^{8}$ Explica Foucault (2000, p. 75) que este proceso ocurre de la siguiente manera: en primer lugar la disciplina analiza, descompone los individuos, tiempos, lugares, gestos, actos, operaciones; en segundo lugar, clasifica los elementos identificados en función de objetivos determinados (ej. ¿cuáles son los niños más aptos para alcanzar un determinado resultado?); en tercer lugar, se establecen las secuencias o coordinaciones óptimas; en cuarto lugar, fija los procedimientos de adiestramiento y de control y por último distingue entre quiénes son ineptos y los demás.

${ }^{9}$ En este caso, lo normal es lo primero y la norma se fija a partir del estudio de las normalidades. Foucault (2000, p. 76 y ss.) lo explica a partir de una epidemia de viruela en el siglo XVIII.

${ }^{10}$ Para la realización de este análisis ha sido esclarecedor el trabajo de Galak (2012).
} 
que resultaban para la acción educativa ${ }^{11}$. Las acciones intervienen el espacio escolar (edificios, muebles), el tiempo escolar (recreos, distribución, suspensión de alumnos enfermos con estricta fecha de reintegro según cada enfermedad), los hábitos higiénicos (vacunación y revacunación obligatoria, aseo, baños, paseos al aire libre, alimentación, sueño, botiquín escolar, ejercicios físicos $)^{12}$. Estos son ejemplos de normación: se descomponen los espacios, los tiempos, los gestos, los actos, las operaciones y luego se clasifican para modificarlos. Las disciplinas producen distribuciones espaciales, temporales, vigilancia, gestos, a partir del adiestramiento progresivo. La revisión de las sesiones del CME en sus inicios muestra como cada aspecto de la vida escolar quedaba bajo la lupa de este Cuerpo ${ }^{13}$, siendo un indicador de la normalización disciplinaria.

Los médicos miembros del CME debían realizar "visitas de inspección higiénica" a las escuelas durante su horario de funcionamiento en el departamento de Montevideo. En el interior del país — “ciudades, villas y pueblos en la República” (Reglamento, 1910, p. 1082) — las visitas serían realizadas por delegados designados que colaboraban en forma honoraria. Durante esas visitas se debía revisar el estado de salud de los alumnos, en particular de los “delicados, enfermizos o anormales" (Reglamento, 1910, p. 1086).

En las visitas higiénicas se completaban fichas sanitarias con la información de los alumnos con la intención de utilizar su información para prevenir enfermedades y para detectar a

\footnotetext{
${ }^{11}$ Según el Reglamento de 1910 (pp.1081-1082), el asesoramiento que el CME debía brindar abarcaba diversos asuntos: estado de salud de los alumnos de las escuelas y construcción de "fichas sanitarias"; reconocimiento individual anual del magisterio; proyectos de edificios escolares atendiendo a ubicación, orientación, ventilación, calefacción, etc.; condiciones higiénicas en que se encuentran los locales escolares y mejoras a realizarles; material de enseñanza; mobiliario escolar; sistema de escritura más conveniente para la salud de los niños; duración del trabajo escolar; horarios y programas de enseñanza; "disposiciones y mejoras de orden higiénico que sean convenientes al escolar, dentro y fuera de la Escuela, así como también los medios más conducentes a la instrucción de atrasados mentales" (p. 1081); sistemas de gimnasia más oportunos; redacción de cartillas para el uso de Magisterio con síntomas de afecciones contagiosas; solicitudes de licencia por enfermedad del personal docente y certificados de buena salud para exámenes y concursos; expedientes de jubilaciones y pensiones escolares; vacunación y revacunación de alumnos y personal docente; conferencias a Personal enseñante sobre higiene escolar; consultorio con horario establecido.

${ }^{12}$ Para ampliar algunos temas, que aquí no se abordan, se recomienda un artículo de Conde, Darrigol y Páez (2018) sobre las significaciones en torno a la arquitectura escolar y los sujetos de la educación en la configuración del CME. Asimismo, se recomienda el trabajo de Espiga (2015).

${ }^{13}$ Se presentan a continuación dos ejemplos. Un extenso informe de Arturo Berro (1908) sobre la recomendación del uso de fuentes sanitarias para proveer agua para beber en las escuelas ya que el uso de jarrito individual no había podido ser establecido y compartir jarritos resultaba peligroso por los potenciales contagios de enfermedades. Sebastián Rodríguez (1909) presentó un proyecto — que fue aprobado- para instituir paseos y excursiones escolares en las escuelas públicas.
} 
los estudiantes anormales e intervenir en su desarrollo. Analizando el Cuerpo Médico Escolar en Argentina, Pablo Scharagrodsky plantea:

La antropometría utilizada en la escuela fue una máquina de clasificar y jerarquizar cuerpos (...). Esta concepción del cuerpo, cuyas huellas hasta cierto punto hoy perduran, sometió las diferencias sociales y culturales a la primacía de lo biológico, o mejor dicho de un imaginario biológico, naturalizando las desigualdades de condiciones y justificándolas a través de observaciones supuestamente científicas (Scharagrodsky, 2007, p. 9).

En este caso la normalización toma en cuenta a la población escolar y la intervención atiende a esta escala, se trata de biopolíticas; se construyen las novedosas nociones de caso, riesgo, peligro y crisis; ya no alcanza con la idea de anular la enfermedad en donde se presenta e impedir el contacto entre enfermos y sanos, explica Foucault (2000, p. 82). Para atender a las situaciones de niños que no encajaban con el patrón de normalidad de los cuerpos se crearon las escuelas para retardados mentales ${ }^{14}$, el Instituto de sordomudos ${ }^{15}$, las clases diferenciales, las Escuelas al Aire Libre ${ }^{16}$ y la colonia marítima de Buceo ${ }^{17}$.

Un ejemplo del funcionamiento de la normalización es el caso de las Escuelas al Aire Libre tal como analiza Espiga (2015, p. 143): “El CME registró que los asistentes mejoraban su condición física: aumentan de peso, talla y el perímetro torácico así como la capacidad respiratoria", además de la coloración del rostro y el carácter del niño según testimonio de Abel Pérez que recoge esta autora.

\footnotetext{
14 Schiaffino (1928, pp.134-135) relata el proceso que se siguió para llegar a un plan sobre la enseñanza de retardados.

${ }^{15}$ Se crea por Ley en 1910 con cuatro maestras que habían sido becadas para estudiar en Buenos Aires. El inspector nacional Abel J. Pérez celebra la conquista de “institutos de anormales” para nuestro país (Pérez, 1910).

16 Alberto Marroche escribe en 1914 un Informe sobre la actuación del CME en su carácter de director de dicho Cuerpo. En él se relata el éxito de la primera de estas Escuelas al Aire Libre, creada en 1913 en el robustecimiento de los niños débiles y propensos a la tuberculosis (Marroche, 1914, págs. 258-263). La creación de estas escuelas al aire libre para atender a niños débiles había sido propuesta por Eduardo Rogé (1910) a partir del estudio de estas experiencias en otras partes del mundo.

17 Sebastián B. Rodríguez solicitó la creación de escuelas marítimas de playa con instalaciones volantes en el Informe del CME de 1925. Schiaffino (1928, p. 136) sostiene que el CME insiste con "las escuelas de playa para débiles" desde 1914 y vuelve a proponer su creación. Finalmente, se crea la Colonia Marítima Escolar del Buceo en 1929.
} 
Los conocimientos de Higiene se incluían en el currículo, como se dijo. Se publicaron libros de texto para alumnos de escuelas públicas y entre ellos destacan los conocimientos de higiene en los manuales de Anatomía, Fisiología e Higiene de Gerardo Olivet Ros (1918) y el manual de Economía Doméstica de María Vidal de Olivet (1918) en el que se detalla cómo elegir, distribuir, amueblar, limpiar y ventilar la casa, cómo conservar y preparar alimentos y los cuidados con el agua para beber, entre múltiples contenidos más, distribuidos cuidadosamente por grado.

La generización de las acciones higiénicas se evidencia en que las tareas de preparación de comidas, limpieza, lavado y planchado de ropa, cuidado de niños, son asignadas al ama de casa. Pero el manual, adecuándose a nuevas feminidades de principios del siglo $\mathrm{XX}$, advierte a las mujeres que no deben descuidar su intelecto. Para eso debían leer revistas, diarios, libros o dedicarse al estudio de la música, pintura o dibujo, ocupaciones que además podrían transformarse en medio de vida en caso de necesidad.

La definición de feminidad a la que se ajusta el Manual de Vidal de Olivet reconoce para las mujeres el ejercicio del intelecto - frente a posturas de la época que incluso cuestionaban la capacidad intelectual de las mujeres - pero las sigue sujetando a las tareas domésticas (Sosa, 2018). La normalización alcanza a las feminidades y masculinidades, se señala cómo ser mujer en estos manuales y se pretende la adecuación a esas normas. Scharagrosky (2007, p. 9) plantea que "los cuerpos masculinos y femeninos fueron, en parte, los efectos más materiales de estas disciplinas escolares" y el currículo fue uno de los mecanismos de producción de género.

Los cuerpos son objeto de saber, como evidencian los temas curriculares que se abordan, pero además son objeto de revisión diaria. Los maestros debían agregar a la revista de limpieza existente desde fines del siglo XIX la observación minuciosa para detectar cualquier sospecha de síntomas. La revisión de limpieza se realizaba en manos, cuello, orejas, cara y boca ${ }^{18} \mathrm{y}$ constituye un mecanismo disciplinario de descomposición de los sujetos para clasificar, fijar procedimientos de adiestramiento de hábitos de limpieza personal en este caso y control de su realización.

\footnotetext{
18 Paulina Luisi (1907), antes de la creación del CME, publicó unos “Apuntes que podrán servir a los aspirantes a maestros para el examen de higiene doméstica”. Este material, con la sintomatología de las enfermedades más comunes y cómo reconocerlas, se identificaba como "Bolilla número 24". El magisterio se preparaba para la vigilancia sanitaria de primer momento. Posteriormente, el CME se encargó de publicar Cartillas que colaboraran con esta tarea.
} 
El CME intervino en las decisiones sobre suspensión de clases debido a epidemias. Este es un ejemplo de biopolíticas, ya que se toman en cuenta los datos de distribución de las enfermedades a nivel de población para sostener las decisiones que se proponen. Consultado en 1909 sobre la posible clausura de una escuela por estar ubicada en un barrio con presencia de viruela, el Cuerpo explica que no es necesario el cierre si se han adoptado las prescripciones de vacunación y revacunación. En ese mismo año, 1909, se produce una epidemia de escarlatina. El diario El Siglo propuso el cierre de escuelas ante la epidemia, pero el CME no estaba de acuerdo con la medida: sostenía que se podía enfrentar con una vigilancia de los posibles casos afectados y con especificaciones para la limpieza de las escuelas dada la poca malignidad de la epidemia (Sesión 5/6/1909). Pocos días después, ante la insistencia de las autoridades educativas, el CME revisa la decisión aconsejando la clausura de escuelas por un tiempo no menor a 40 días. Se fundamenta dicha decisión para dar satisfacción "a la opinión pública alarmada por la propaganda de la prensa" (Sesión 22/6/1909).

La epidemia de la gripe ("grippe”) en Montevideo, con dos olas en 1918 y 1919, trae nuevamente la discusión sobre la medida de clausura de las clases escolares. En estas oportunidades el CME tampoco estaba de acuerdo con dicha medida porque la gripe no mostraba tendencia a afectar mayormente a la población infantil. No se consideraba la posibilidad de que el alumnado se transformara en vehículo de contagios a mayor escala. De todas formas, las escuelas fueron cerradas por las autoridades sanitarias, quizá con algo de retraso, en 1919 porque hubo una "verdadera hecatombe (de) atacados en el Magisterio" (Schiaffino, 1925, p. 299).

Puede discutirse la incidencia efectiva de las intervenciones reseñadas en las escuelas, punto que se plantea brevemente en el siguiente apartado.

\section{Alcance de las acciones del Cuerpo Médico Escolar}

El alcance de las aspiraciones higienistas ha sido objeto de apreciaciones muy distantes. Barrán (1993) expresa:

El higienismo dominante en el Novecientos fue una de las más exitosas empresas de control social jamás imaginadas por cuanto se sirvió de una obsesión que ya era colectiva — la salud y la vida larga-, para propagar e imponer cambios funcionales a la "modernización" de la sociedad (pp. 14-15). 
Por el contrario, Reyes Möller (1996) plantea:

La limitada aplicación práctica del discurso higienista es una muestra notoria de cómo estas tendencias podían darse de plano contra la realidad de buena parte de los educandos y del propio medio escolar. Respecto de las normas higiénicas, la Escuela Pública no predicaba con el ejemplo, sometiendo a sus párvulos a una situación de hacinamiento en muchos casos similar a la vivida en sus hogares (p. 234).

Por su parte, Espiga plantea la "incidencia del discurso higienista en las prácticas de aula, no solo aproximando e instruyendo en nuevas nociones de higiene, anatomía o fisiología, sino también impactando en las formas de enseñanza" (2015, pp. 192-193). La autora analiza la tríada obligatoriedad, asistencia irregular y analfabetismo como la "tríada a resolver", la asistencia a la escuela fue imperativa, no sólo para "abatir el analfabetismo, instruir en nuevos valores o fundar una nacionalidad. La escuela, por su alcance universal y genérico, permitió implementar y ensayar políticas preventivas de salud pública” (Espiga, 2015, pp. 19-20).

La incidencia del CME también pudo verse en la construcción de edificios escolares de la época ${ }^{19}$ y en las refacciones que se fueron haciendo en edificios más antiguos tal como plantean Conde, Darrigol y Páez (2018).

Una pregunta pertinente es cuál fue la cobertura de las visitas higiénicas a escuelas. Los datos que se presentan a continuación muestran que tuvieron un alcance considerable en el período relevado. Los Informes del CME presentan las visitas higiénicas realizadas por médicos y médica del CME para el departamento de Montevideo, tal como era su competencia. En 1914 las escuelas públicas de Montevideo eran 103 y se realizó el fichaje de estudiantes en 56 de ellas (Pérez, 1916); en el quinquenio 1915-1919 se cubrían 2/3 de las 101 escuelas que había en el departamento (Schiaffino, 1925, p. 282); a partir de 1920 se cubre el total de escuelas públicas de Montevideo (su número asciende a 108 en 1924), cobertura que significa 1/5 del alumnado (Schiaffino, 1925, p. 282); en 1925 la cantidad de escuelas visitadas se reduce a 70, se aduce un crecimiento de la población escolar (Rodríguez, 1925); en 1926 se cubre la totalidad de escuelas públicas montevideanas nuevamente (Schiaffino, 1927); en 1927 se visitan 139 escuelas que no

\footnotetext{
${ }^{19}$ La Memoria 1911-1914 (Pérez, 1916) muestra fotografías de las espléndidas escuelas construidas en la etapa. Amplias, para mantener distancia social, con posibilidades de ventilación y ajustadas a los preceptos higienistas para locales escolares.
} 
constituyen la totalidad de escuelas públicas del departamento pero se agregan además visitas higiénicas a escuelas privadas (Schiaffino, 1928).

Sin duda, se podría seguir incrementando este listado, pero en este trabajo el objeto de atención pasará a ser la normalización del magisterio.

\section{La normalización de maestras, maestros y estudiantes magisteriales: "velando por la depuración del futuro magisterio"}

En este apartado se presenta la normalización del magisterio. Se plantea en primer lugar el currículo para dar paso luego a las acciones del CME en el magisterio a partir de dos mecanismos: en primer lugar, la selección de aspirantes a estudiar magisterio y a concursar por cargos y, en segundo lugar, el reconocimiento anual del estado de salud del magisterio que podía derivar en la cesación temporal o definitiva de la continuidad como docente.

\section{El currículo: higienismo y eugenesia}

El currículo para estudiantes magisteriales incorporó Higiene y Eugenesia. Dogliotti (2012, p. 121) presenta los contenidos de un programa adoptado provisionalmente en 1910 proyectado por la Dirección del Instituto Normal de Maestros, agrupando las asignaturas en relación con el área temática. Uno de los grupos temáticos de asignaturas organizados por esta autora es: Historia Natural, Anatomía y Fisiología, Higiene, Higiene Escolar, Economía Doméstica, Maternología y Labores del Hogar. Pero la Higiene no sólo se configura como asignatura, sino que también está contenida en otras asignaturas: "El higienismo es la nueva moral laica del Novecientos y se presenta regulando desde los conocimientos emanados de la medicina la mayoría de las asignaturas del programa" (Dogliotti, 2012, p. 126). Por otro lado, las asignaturas como Economía Doméstica y Maternología y, como demuestra Dogliotti, la educación del cuerpo prevista, muestran la generización femenina del currículo, es decir, una normación determinada para la feminidad.

En cuanto a la eugenesia, según Barrán (1999), en 1925 se implantó en los institutos normales una Cátedra de Higiene Infantil dictada por el pediatra Américo Mola que incluía contenidos propios de la eugenesia. En 1932 se publicó un texto de estudio de esta Cátedra que se utilizaba en la formación magisterial. Otra cátedra que incorporó una unidad curricular sobre 
Eugenesia fue la Cátedra de Higiene Social para estudiantes normalistas creada por propuesta de la Dra. Paulina Luisi, integrante del CME, que comenzó a dictarse en 1926. Cuando Luisi tomaba licencia en el dictado de la Cátedra, la sustituía el Dr. Rafael Schiaffino ${ }^{20}$ (también integrante del CME). Aun así, a la interna del CME hubo disidencias. Esto se advierte en los comentarios de Luisi sobre el final de la Cátedra hacia el año 1930 debido a que generaba resistencias entre las autoridades normalistas y a que el director del CME del momento había calificado como "inútil” a dicha Cátedra (Luisi, 1950: 265). Las fuentes consultadas no permiten conocer el origen de las diferencias de criterios entre miembros del CME, si acaso se vinculaban con la incorporación que Luisi había realizado de contenidos de educación sexual en la Cátedra mencionada o si la discordancia alcanzaba a otros temas. Lo cierto es que la Eugenesia fue incorporada al currículo y también formaba parte de conferencias anexas a la Cátedra dictadas por Luisi para el magisterio en ejercicio y público en general (Luisi, 1950).

\section{Selección de aspirantes a estudiar magisterio y concursar: una biopolítica de exclusión}

El CME fue el encargado de la selección de aspirantes a estudiar magisterio ${ }^{21}$ y a dar el concurso para ocupar cargos en el magisterio ${ }^{22}$. También en este caso puede seguirse el pasaje de preocupaciones higiénicas — como la necesidad de apartar a maestros enfermos para evitar el contagio de distintas enfermedades en las escuelas - a una selección realizada con motivos que se adentran en los discursos eugenésicos ${ }^{23}$. Para Reggiani (2014, p. 57), "la medicina ya no se conformaba con la mera preservación de la salud sino con la optimización del rendimiento psicofísico individual. Probablemente sea aquí donde debamos buscar el legado más persistente e insospechado de la utopía eugenésica". Ciertamente, la búsqueda de la "depuración” que se anuncia en el título de este apartado, que proviene de una expresión de Sebastián B. Rodríguez

\footnotetext{
${ }^{20}$ Dato relevado en el Archivo de los Institutos Normales de Montevideo.

${ }^{21}$ En ese momento existían sólo dos instituciones para estudios magisteriales: el Instituto de Señoritas y el Instituto de Varones, ambos situados en Montevideo. Sobre esta formación ver el trabajo de Luis Delio (2009).

22 Había dos maneras de desempeñarse como maestra/o: cursando la formación en los institutos normales — maestros normalistas - o dando examen para acceder a cargos — maestro nacional (Archivo de los IINN).

${ }^{23}$ Este planteo es similar al realizado por Rodríguez (2016) cuando analiza la selección de aspirantes al curso de profesores de educación física realizada por la Oficina Médica de la Comisión Nacional de Educación Física en 1924: "La medicina preventiva, el higienismo, será uno de los discursos predominantes entre los estadistas del novecientos. A su preocupación por prevenir y 'atacar' las enfermedades, se le entrelaza otra muy asociada a los discursos eugenistas, la necesidad de predecir el desarrollo normal de los alumnos y que las debilidades orgánicas de 'los defectuosos' no entorpezcan el progreso de la raza' (p. 214).
} 
(1925), muestra cómo influyeron estos discursos en la construcción de una "normalidad" y una "otredad" que van bastante más allá que las preocupaciones higienistas de preservación de la salud.

Los motivos por los cuales se concedía o se negaba el acceso a la carrera del magisterio fueron modificándose con el tiempo. Un extenso informe de 1914 sobre la acción del CME elaborado por quien lo presidía en ese momento, el Dr. Luis Marroche, consigna que se realizaba un examen médico individual de alumnos de los Institutos Normales de Varones y Señoritas. Para el ingreso a dichos institutos se exigía "un certificado que acredite su buen estado de salud y la ausencia de defectos físicos que lo inhabiliten para el ejercicio del magisterio, documento expedido por el Cuerpo Médico Escolar" (Marroche, 1914, p. 195). Este certificado obligatorio para los estudiantes alcanzaba también a quienes quisieran concursar por el cargo de maestro nacional. Es decir, antes de las dos modalidades de ejercer el magisterio, como normalista o nacional, había que enfrentar el examen de selección, en el caso de normalistas previo a los estudios y en el otro caso previo al concurso.

El certificado de salud que se exigía a aspirantes a estudiar magisterio y a concursantes por cargos en el magisterio, generó resistencias entre las personas denegadas. El Poder Ejecutivo intervino en estas disputas, decretando que se concediera una nueva revisión a los maestros declarados impedidos por el CME e interponiendo la opinión del Consejo Nacional de Higiene en los casos en disputa. Marroche (1914) estimó que dicha resolución resultaba inconveniente porque las conclusiones del CME y del Consejo Nacional de Higiene podían contradecirse ya que el criterio de aceptación del CME era más estricto; mientras que el Consejo Nacional de Higiene aceptaba a algunos pretuberculosos o tuberculosos incipientes, el CME determinaba que:

el esfuerzo, privaciones y fatigas que exige el ejercicio del magisterio o los desvelos y desgastes nerviosos que requiere el estudio para conseguir el título de maestro, compromete en un porvenir casi nunca muy lejano, la salud de aquel, constituyendo entonces un serio peligro para las personas que lo rodean, especialmente para los niños que están en contacto directo con este; constituye para el Estado una causa de recargo en su presupuesto por las frecuentes y prolongadas licencias que hay forzosamente que aconsejar, y pedagógicamente, 
porque interrumpe la buena marcha de la enseñanza con los cambios frecuentes de maestros en una clase (Marroche, 1914, pp.196-197).

Marroche continuó la defensa de las decisiones del CME en la exclusión de postulantes a estudiar magisterio o a concursar, sosteniendo que los certificados expedidos por enfermedad a maestras/os en ejercicio mostraban la alta incidencia de las afecciones del aparato respiratorio (25\%), particularmente por tuberculosis, por lo que proponía el examen anual del magisterio de carácter obligatorio y algunas medidas a tomar en caso de detectar tuberculosis. Su argumento era que la rigurosidad en los exámenes médicos en la admisión de candidatos al magisterio, permitiría eliminar la incidencia de esta enfermedad. Por este motivo, el CME solicitó la derogación o anulación del derecho de los docentes o estudiantes denegados de apelar ante una autoridad distinta al CME. El CME logró que se lo considerara como único Tribunal de alzada. La resolución de la Dirección General del Instrucción Primaria, firmada por Abel J. Pérez, en la que se concedió lo solicitado al CME, fundamenta la decisión de la siguiente manera:

el Cuerpo comienza su tarea con el simple aspirante a ingreso a los institutos, o a la iniciación de los exámenes para maestros nacionales, y la continúa hasta los maestros que en pleno funcionamiento contraen enfermedades contagiosas, lo que tiene que hacer obligatoriamente en el primer caso para evitar que emprendan, inútilmente para ellos mismos, la carrera aquellos aspirantes que no resistirán, notoriamente, ni a las tareas de preparación y mucho menos las muy exigentes requeridas para el ejercicio de la profesión, en el caso de que llegasen a obtener el diploma (Pérez, citado por Marroche, 1914, p. 199).

El contenido del examen médico individual ${ }^{24}$ de los alumnos de los Institutos Normales de Varones y de Señoritas fue extendiéndose ya que en 1915 se incorporó al CME un oculista y un otorrinolaringólogo ("rino-oto-laringólogo"), además de una nurse para auxiliar a las mujeres aspirantes al magisterio con los exámenes de orina (Marroche, 1914).

Los problemas respiratorios, especialmente la tuberculosis, justificaron las acciones del CME en la exclusión de docentes en el episodio reseñado. Pero, como se verá, los patrones de normalidad para el magisterio propulsados por el CME iban más allá.

\footnotetext{
${ }^{24}$ En este examen se revisa: piel y cuero cabelludo, sistema linfático, aparato visual, aparato digestivo y anexos, faringe, lenguaje articulado, aparato respiratorio, aparato circulatorio, esqueleto (Marroche, 1914, pp. 201-202).
} 
Los maestros no deben ser para los alumnos un peligro, sino una garantía que resida no sólo en sus cualidades morales e intelectuales, sino también en un hábito exterior sano y robusto, y la utilidad pedagógica de una buena constitución ha preocupado ciertamente a aquellos que están encargados de asegurar la educación de la juventud (Marroche, 1914, pp. 240-241).

Al igual que en las fichas sanitarias de escolares, se incorpora además de la enfermedad, la posibilidad de un enfermedad futura, por lo que se hacía necesario aceptar a personas con "una buena constitución". El "hábito exterior sano y robusto" refiere a la apariencia. Se utiliza metafóricamente la palabra hábito; lo que se espera es que la apariencia de la persona denote su salud presente y futura. La idea de robustez irá ganando espacio en la consideración del patrón de normalidad del cuerpo de quienes se dedicaban al magisterio, como se verá.

Marroche propuso como guía para los exámenes médicos de estudiantes de magisterio a un médico escolar inglés -Mr. Williamson- quien recomendaba que se rechazara a un candidato si él o ella "no parecen bastante vigorosos para soportar el esfuerzo que necesita la duración de los estudios en la escuela normal, o si presenta los signos de una enfermedad que le impida seguir de una manera continua las tareas del profesorado" (Williamson en Marroche, 1914, p. 241). Concretamente proponía rechazar a personas afectadas por determinadas enfermedades respiratorias, cardíacas, diabetes, neurastenia o histerismo confirmado ${ }^{25}$, anemia, afecciones gástricas, etc. y a esto agregaba defectos que no hay que dejar pasar: de la visión, de la audición o de la fonación. Como se viene haciendo notar, la línea de demarcación ya no era sano/enfermo con enfermedades contagiosas, sino que era sano/enfermo (con enfermedades contagiosas o no), con defectos, sin "apariencia sana o robusta".

Hasta el año 1925 no vuelve a publicarse un informe tan minucioso de las acciones del CME. Este segundo informe, firmado por Rafael Schiaffino (1925), recoge los años anteriores, por lo que la información que contiene es sustancial. Allí se insiste en la evaluación de la totalidad de estudiantes magisteriales: "uno de los objetos preferentes de nuestra atención al hacernos cargo del Cuerpo Médico Escolar, ha sido la vigilancia de los Institutos Normales” (p. 309).

${ }^{25}$ Este punto se analiza más adelante en el texto. 
Schiaffino expresa los criterios para aceptar a estudiantes magisteriales o a concursantes. El primer criterio es el "coeficiente de robustez" (1925, p. 313). Para determinar este coeficiente se utilizaban datos antropométricos tomados por el CME, por promedio se fijaban parámetros y se rechazaba a quien quedara por fuera de dichos parámetros. Formaban parte del coeficiente la estatura, el peso, el perímetro torácico y la desproporción entre esos factores. Se excluía previamente a los casos de anemia, escoliosis y a un "etcétera" al que se refiere, dejando a cargo del médico examinador la exclusión. Otros criterios para excluir estudiantes o concursantes eran la temperatura corporal mayor de $37^{\circ}$, las afecciones cardíacas y las lesiones de vista u oído.

Schiaffino (1925, p. 314) sostenía que no sólo se debía pensar en si el estado de salud perjudicaría el desarrollo de la carrera de estudios de magisterio, también en la posibilidad del desempeño del magisterio dadas ciertas afecciones o defectos. Los listados de motivos de rechazo incluyen deformaciones en el tórax, parálisis del miembro superior derecho, deformación congénita de la boca, entre otros. Se buscaba más que la presencia de enfermedades o incluso las posibles enfermedades futuras; se buscaba una apariencia saludable e incluso armónica (la desproporción de las medidas era motivo de rechazo también). La falta de agudeza visual o auditiva como condición para la aceptación dejaba por el camino a personas con miopía, por ejemplo.

En estas decisiones hay algunas que se ajustan a la normalización disciplinaria porque se establecen normas que señalan de qué lado está lo aceptable y lo no aceptable y otras que se ajustan a la normalización desde las biopolíticas. El caso del coeficiente de robustez es uno de estos, la normalidad que entraña ese coeficiente se establece a partir de parámetros promedio fijados a partir de datos antropométricos tomados por el CME (la normalidad antes que la norma, como explica Foucault en estos casos en los que se miran los datos de la población).

Esta política tuvo detractores, según lo que expresó:

No ha faltado quien manifestara que sólo en las causas de enfermedades contagiosas o desagradables o que impidan material o normalmente el ejercicio de la profesión deben rechazarse los candidatos. Nada más erróneo. Una lesión cardíaca, por ejemplo, (...) sentirá el efecto del trabajo (...) ese estado, fuera de los trastornos que apareja al maestro es una causa de disturbios en la vida de la escuela. La enseñanza irregular primero, las licencias repetidas después, 
perjudican la marcha normal de las aulas y pesan sobre el erario más tarde con la jubilación temprana (Schiaffino, 1925, p. 314).

Convencido de los criterios desarrollados, Schiaffino propuso un Reglamento de certificados de buena salud para que fuera aprobado, cuestión que se resolvió a su favor. Este Reglamento contiene las condiciones para la obtención del certificado (sea estudiante o maestro en ejercicio): vacunas al día, aseo suficiente, sin pediculosis, boca en perfectas condiciones (no se aceptan caries ni siquiera en tratamiento), desarrollo físico ajustado a promedios de su edad, obesidad y desnutrición se descartan, sin defectos ni deformaciones de la cara, sin desviaciones de columna vertebral, sin malformación torácica, se mencionan luego enfermedades por las que se debe negar el certificado (de la visión, de los oídos, de la laringe, de la fonación, nariz, garganta, piel, afecciones cardíacas, pulmonares, glandulares, afecciones del sistema nervioso, diabetes y se agrega un artículo abierto en el que se puedan incorporar afecciones). La lista de motivos de exclusión era extensa y severa, como se puede apreciar.

Un informe posterior en el que se explican acciones similares, firmado por Sebastián B. Rodríguez, defiende la acción del CME de la siguiente manera:

Toda medida nueva y que viene a lesionar intereses, siempre ha levantado resistencias, pero el Cuerpo Médico Escolar, velando por la depuración del futuro magisterio, y también por sus fueros, cree que debe seguirse adelante en el camino emprendido (Rodríguez, 1925, p. 94).

En lo que antecede se mostraron los motivos de exclusión de estudiantes y concursantes, los cambios en sus justificaciones y las resistencias que despertó. Pero las trabas no estaban solo en el ingreso; también se podía perder el cargo docente, como se mostrará en el siguiente tramo.

\section{Reconocimiento anual del estado de salud del magisterio}

El reconocimiento anual del estado de salud del magisterio comenzó realizándose por citación a docentes de Montevideo al consultorio y desde 1919 se definió su realización en las escuelas en la misma oportunidad en que se realizaran las fichas sanitarias al alumnado. El Informe de 1914 (Marroche, 1914) revela que de 698 docentes citados acudieron 564 y un 29\% de dichos docentes en ejercicio estaban más o menos enfermos. La mayoría de las afecciones correspondían a las vías respiratorias (89\%). Nuevamente, la alta incidencia de enfermedades 
respiratorias fue el fundamento de las políticas desarrolladas. El Informe de 1924 (Schiaffino, 1925) hizo foco en la incidencia de la caries dental sin corregir en el magisterio que alcanzaba al 20\%. Para los aspirantes a magisterio y a dar concurso de maestros nacionales se exigía la completa higienización bucal, por lo que el CME, ante los datos recogidos, solicitó que para ejercer el magisterio se exigiera lo mismo. No fue concedida esta petición que habría removido de sus cargos a un quinto del magisterio, pero Schiaffino celebraba que como la exigencia aplicaba para aspirantes a estudiar magisterio y al concurso, a la larga un mayor porcentaje de maestros tendría salud bucal.

Los motivos de cese - temporal o definitivo - a partir de los dictámenes del CME son similares a los que se esgrimen para excluir a estudiantes y concursantes, por lo que no se volverán a plantear aquí salvo el caso de las exclusiones por histerismo que merecen atención. Según Barrán y Nahum (1990, p. 76) en el Uruguay del Novecientos hubo un "auge de la histeria". La histeria es más una estructura que una enfermedad y entronca en la sexualidad de la época (Chauvelot, 2001). Así lo explica Scharagrodsky para fines del siglo XIX en Argentina:

Si la mujer sana fue aquella que aprendió el difícil oficio de la maternidad respetando los cánones morales comúnmente aceptados sobre la femineidad, no desviándose de la elección sexual heteronormativa, no cuestionando las relaciones de poder, la división "sexual" del trabajo y la división de tareas y no convirtiéndose en políticamente disidente; las mujeres enfermas fueron aquellas que no aceptaron estos mandatos sociales y culturales. (...) [Entre las enfermedades] la que primó en el discurso médico referido a las mujeres fue la histeria (Scharagrosky, 2011, pp. 104-105).

La construcción de feminidades y sexualidades en el Novecientos, así como la intervención del poder y saber médicos en estas construcciones a partir de la histeria y su tratamiento fueron reseñadas por Barrán (1992). Y este, que es un analizador de las construcciones de feminidad y sexualidad del Novecientos, constituía otro de los motivos de exclusión de la carrera magisterial. 


\section{Líneas de cierre}

En este artículo se presentaron las líneas de acción del CME y sus fundamentos. La intervención del discurso médico en múltiples aspectos de la vida de las escuelas de principios de siglo XX en el Uruguay es una muestra del proceso de medicalización de la sociedad: el discurso médico incide en el discurso pedagógico.

Se revisó la incorporación de contenidos higienistas en el currículo y se señaló la generización de las acciones higiénicas y la consiguiente generización del currículo. Cuantiosos aspectos de la vida en las escuelas, como el uso del espacio, del tiempo escolar, los hábitos higiénicos, quedaron bajo la vigilancia sanitaria. Esta vigilancia tuvo como responsables a los docentes —en la revisión de aseo diaria y en el aviso al CME frente a la sospecha de enfermedad - y al CME con sus visitas higiénicas y sus campañas de higiene en las escuelas (cartillas, folletos, informes). El CME intervino en las discusiones sobre biopolíticas frente a situaciones de enfermedades contagiosas, aconsejando o desaconsejando la clausura de cursos, la suspensión de parte del alumnado y el confinamiento en casos de enfermedad.

Las fichas sanitarias a escolares realizadas por el CME alcanzaron al departamento de Montevideo, aun así, contribuyeron a la normalización de la niñez. La debilidad generaba sospechas de enfermedad futura frente a la que había que intervenir. La consideración de parte de la infancia como "anormal" y la creación de circuitos de escolarización segregados forma parte de este período.

En el artículo se hizo foco en la normalización del magisterio. Particularmente, la acción del CME es relevante en los Institutos Normales de Montevideo por la selección de aspirantes a estudiar magisterio y de concursantes por cargos docentes. En el período relevado se realizaba el reconocimiento anual del estado sanitario del magisterio, en el departamento de Montevideo. Los significados de los exámenes de selección o de vigilancia del estado sanitario del magisterio pueden identificarse con ideas higienistas que se superponen con otras de corte eugenésico.

Los cuerpos de docentes debían ser completamente sanos pero la "depuración" del magisterio abarcaba más. Los cuerpos debían ser robustos y armónicos —ajustándose a un "coeficiente de robustez"-, de apariencia sana y sin dejar lugar para la duda de la posible presencia de enfermedades en el futuro, sin obesidad ni desnutrición y sin defectos. Más allá de los dichos de integrantes del CME, ¿cuánto influyeron estos patrones de normalidad en el 
magisterio? En estudiantes y concursantes fue determinante porque, en la selección, se excluye. En el magisterio en general también hubo incidencia, acotada al departamento de Montevideo, en la medida en que a partir del reconocimiento anual del magisterio se decidía la suspensión temporal o definitiva de los cargos. Hubo resistencias a estas medidas, algunas registradas como la protesta por los resultados de los exámenes llevados adelante por el CME que se reseñó en este trabajo - y otras que pueden inferirse a partir de la defensa y las múltiples justificaciones del enfoque que el CME realiza.

\section{Referencias}

Armús, D. (2016). Eugenesia en Buenos Aires: discursos, prácticas, historiografía. História, Ciências, Saúde Manguinhos, 23(10), 149-169.

Barrán, J. P. (1992). Medicina y sociedad en el Uruguay del Novecientos. 1. El poder de curar. Montevideo, Uruguay: Ediciones de la Banda Oriental.

Barrán, J. P. (1993). Medicina y sociedad en el Uruguay del Novecientos. 2. La ortopedia de los pobres. Montevideo, Uruguay: Ediciones de la Banda Oriental.

Barrán, J. P. (1995). Medicina y sociedad en el Uruguay del Novecientos. 3. La invención del cuerpo. Montevideo, Uruguay: Ediciones de la Banda Oriental.

Barrán, J. P. (1999). Biología, Medicina y eugenesia en Uruguay. Asclepio, 51(2), 11-50.

Barrán, J. P. (2011). Historia de la sensibilidad en el Uruguay. Montevideo, Uruguay: Ediciones de la Banda Oriental.

Barrán, J. P. y Nahum, B. (1990). Batlle, los estancieros y el imperio británico, tomo I El Uruguay del Novecientos ( $2^{\mathrm{a}}$ ed.). Montevideo, Uruguay: Ediciones de la Banda Oriental.

Castro, E. (2004). El vocabulario de Michel Foucault. Bernal, Argentina: Universidad Nacional de Quilmes.

Conde, S., Darrigol, M. y Páez, S. (2018). Configuración del Cuerpo Médico Escolar en el Uruguay (1903-1915) Significaciones en torno a la arquitectura escolar y los sujetos de la educación. Didáskomai, 9, 1-17.

Chauvelot, D. (2001). Historia de la histeria. Sexo y violencia en lo inconsciente. Madrid, España: Alianza.

Darré, S. (2005). Políticas de género y discurso pedagógico. La educación sexual en el Uruguay del siglo XX. Montevideo, Uruguay: Trilce.

Delio, L. (2009). Historia de la formación docente. La enseñanza normal nacional. Montevideo, Uruguay: Cruz del Sur.

Dogliotti, P. (2012). Cuerpo y currículum: discursividades en torno a la formación de docentes de educación física en Uruguay (1874-1948) (Tesis de Maestría). Universidad de la República, Montevideo, Uruguay.

Espiga, S. (2015). La infancia normalizada. Libros, maestros e higienistas en la escuela pública uruguaya 1885 1918. Montevideo, Uruguay: Antítesis Editorial. 
Facal, S. (2002). Política inmigratoria de puertas cerradas. Uruguay frente a la llegada de refugiados españoles republicanos y judíos alemanes. Revista Complutense de Historia de América, 28, 169-183.

Foucault, M. (1996). Vigilar y castigar. Nacimiento de la prisión (24a ed.). México D. F., México: Siglo XXI (Trabajo publicado originalmente en 1975).

Foucault, M. (2000). Defender la sociedad. Buenos Aires, Argentina: FCE.

Galak, E. (2012). Del dicho al hecho (y viceversa). El largo trecho de construcción del campo de la formación profesional de la Educación Física en Argentina (Tesis de posgrado). Universidad Nacional de La Plata, Argentina.

Lavrin, A. (2005). Mujeres, feminismo y cambio social en Argentina, Chile y Uruguay 1890-1940. Santiago de Chile, Chile: Ediciones de la Dirección de Bibliotecas, Archivos y Museos.

Lionetti, L. (2011). Discursos, representaciones y prácticas educativas sobre el cuerpo de los escolares. Argentina en las primeras décadas del siglo XX. Cuadernos de Historia, 34, 31-52.

Reggiani, A. (2014). Eugenesia y cultura física. Tres trayectorias históricas: Francia, Gran Bretaña, Argentina. En P. Scharagrodsky (comp.), Miradas médicas sobre la cultura física. Argentina 1880-1970 (pp. 17-58). Buenos Aires, Argentina: Prometeo.

Reggiani, A. (2019). Historia mínima de la eugenesia en América Latina. México D. F., México: El Colegio de México.

Reyes Möller, C. (1996). Del empaque y el desenfado corporal en el Novecientos. En J. P. Barrán, G. Caetano y T. Porzecanski (dirs.), Historias de la vida privada en el Uruguay. El nacimiento de la intimidad 1870-1920. V. II (pp. 231-252). Montevideo, Uruguay: Taurus.

Rodríguez, C. (2016). El examen de ingreso al curso de Profesores de Educación Física en el Uruguay: una herramienta de selección en clave biotipológica. Cartografías del Sur, 4, 196-223.

Sapriza, G. (1988). Paulina Luisi. En G. Sapriza, Memorias de rebeldía. Siete historias de vida (pp. 77-107). Montevideo, Uruguay: Puntosur.

Sapriza, G. (2008). La hora de la eugenesia: las feministas en la encrucijada. En I. Morant (dir.), Historia de las mujeres en España y América Latina III del siglo XIX a los umbrales del XX (pp. 889-913) (2 $2^{\mathrm{a}}$ ed.). Madrid, España: Cátedra.

Sapriza, G. (2011). Historia de la (des)penalización del aborto en Uruguay. "Aborto libre": la corta experiencia uruguaya (1934-1938). En N. Johnson, G. Sapriza, A. López Gómez, C. Pallas, A. Castro, O. Sarlo, C. Moreira, y M. A. Andreoli, (Des)penalización del aborto en Uruguay: prácticas, actores y discursos (pp. 19-64). Montevideo, Uruguay: Universidad de la República.

Scharagrodsky, P. (2007). El cuerpo en la escuela. Buenos Aires, Argentina: Ministerio de Educación, Ciencia y Tecnología.

Scharagrodsky, P. (2011). Cuerpos femeninos en movimiento o acerca de los significados sobre la salud y la enfermedad a fines del siglo XIX en la Argentina. Pro-Posições, 22(3), 97-110. 
Sosa, F. (2018). Acceso de mujeres a la educación secundaria pública en el Uruguay (1911-1936): femineidades, sexualidades y maternidades (Tesis de Maestría). FLACSO. Sede Académica Argentina. Buenos Aires, Argentina.

Stepan, N. (1991). The hour of Eugenics. Race, Gender and Nation in Latin America. Ithaca \& London: Cornell University Press.

\section{Fuentes}

Berro, A. (1908). Informe expedido por el doctor Berro en un expediente iniciado por el Director de la Escuela de $2^{\circ}$ grado número 13 de la Capital, solicitando que se dispusiera la construcción de un soporte para jarritos. Anales de Instrucción Primaria, Año VII, Tomo VI. Montevideo, Uruguay: El Siglo Ilustrado, pp. 240-249.

Cuerpo Médico Escolar. (1909). Reglamentación para el ejercicio de sus funciones de 7 de agosto de 1908. Anales de Instrucción Primaria, Año VII, Tomo VI. Montevideo, Uruguay: El Siglo Ilustrado, pp. 207-209

Reglamento del Cuerpo Médico Escolar Nacional, aprobado por resolución gubernativa del 18 de junio de 1910. Anales de Instrucción Primaria, Año IX, Tomo IX, Nos. 7 a 14, julio de 1910 a marzo de 1911. Montevideo, El Siglo Ilustrado, pp. 1080-1090.

Decreto-Ley de Educación Común de 24 de agosto de 1877.

Landeira, M. (1929). Informe del Dr. Manuel Landeira acerca de la Colonia Marítima Escolar del Buceo. En E. Acevedo (1930), Memoria de Instrucción Primaria correspondiente a 1929 (pp. 214-231). Montevideo, Uruguay: Imprenta Nacional.

Luisi, P. (1907). Sintomatología de las enfermedades más comunes. Anales de Instrucción Primaria, Tomo IV, Nos. 1 a 5. Montevideo, Uruguay: El Siglo Ilustrado, pp. 60-65.

Luisi, P. (1916). Algunas ideas sobre Eugenia. Montevideo, Uruguay: El Siglo Ilustrado.

Luisi, P. (1919a). Para una mejor descendencia. Buenos Aires, Argentina: Casa Editora Juan Perrotti.

Luisi, P. (1919b). Higiene del crecimiento. Anales de Instrucción Primaria, Año XVI-XVII, Tomo XVI, Nos. 1 a 3 , enero a marzo de 1919. Montevideo, Uruguay: El Siglo Ilustrado, pp. 39-55.

Luisi, P. (1950). Una Cátedra de Higiene Social. En Luisi, P. (1950). Pedagogía y conducta sexual. Montevideo, Uruguay: El Siglo Ilustrado, pp. 247-315.

Marroche, A. (1914). El Cuerpo Médico Escolar, capítulo VIII. En A. Pérez (1916), Memoria correspondiente a los años 1911 a 1914 de la Dirección General de Instrucción Primaria (pp. 195-284). Montevideo, Uruguay: Barreiro y Ramos.

Olivet Ros, G. (1918). Anatomía, Fisiología e Higiene. Anales de Instrucción Primaria, Año XV-XVI, Tomo XV, nos. 1-12, Julio de 1917 a junio de 1918. Montevideo, Uruguay: El Siglo Ilustrado, pp. 523-618.

Pérez, A. (1907). Apuntes sobre higiene escolar. Anales de Instrucción Primaria, Tomo IV, Nos. 1 a 5. Montevideo, El Siglo Ilustrado, págs. 257-283. 
Pérez, A. (1910). Cuerpo Médico Escolar-Instituto de Sordo-Mudos. Memoria correspondiente a 1908 de la Dirección General de Instrucción Primaria, Tomo II (pp. 309-312). Montevideo, Uruguay: Barreiro y Ramos.

Pérez, A. (1916). Memoria correspondiente a los años 1911 a 1914 de la Dirección General de Instrucción Primaria. Montevideo, Uruguay: Barreiro y Ramos.

Rogé, E. (1910). Las escuelas al aire libre o escuelas selváticas. Anales de Instrucción Primaria, Año VIII, Tomo VIII, Enero-Junio 1910, Nos. 1 a 6. Montevideo, Uruguay: El Siglo Ilustrado, pp. 151-157.

Rodríguez, S. B. (1907). Contribución al desenvolvimiento de la Higiene Escolar en algunos países sudamericanos. Anales de Instrucción Primaria, Tomo IV, Nos. 1 a 5. Montevideo, Uruguay: El Siglo Ilustrado, pp. 24-48.

Rodríguez, S. B. (1909). Proyecto del doctor don Sebastián B. Rodríguez, instituyendo los paseos y excursiones escolares, en las escuelas públicas. Anales de Instrucción Primaria, Año séptimo, Tomo VI, 1909. Montevideo, Uruguay: El Siglo Ilustrado, pp. 251-253.

Rodríguez, S. B. (1925). El Cuerpo Médico Escolar. En E. Acevedo (1926), Memoria de Instrucción Primaria correspondiente a 1925 (pp. 73-94). Montevideo, Uruguay: Imprenta Nacional.

Reglamento del Cuerpo Médico Escolar. (1927). En E. Acevedo (1928), Memoria de Instrucción Primaria correspondiente al año 1927 (pp. 151-155). Montevideo, Uruguay: Imprenta Nacional.

Sesión del 5/6/1909 del CME. Anales de Instrucción Primaria, Año VII, Tomo VI, 1909, Montevideo, Uruguay: El Siglo Ilustrado, p. 233.

Sesión del 22/6/1909 del CME. Anales de Instrucción Primaria, Año VII, Tomo VI, 1909, Montevideo, Uruguay: El Siglo Ilustrado, p. 235.

Schiaffino, R. (1925). Cuerpo Médico Escolar. En E. Acevedo (1925), Memoria de Instrucción Primaria correspondiente al año 1924 (pp. 281-323). Montevideo, Uruguay: Imprenta Nacional.

Schiaffino, R. (1927). Cuerpo Médico Escolar. En E. Acevedo (1927), Memoria de Instrucción Primaria correspondiente al año 1926 (pp. 101-121). Montevideo, Uruguay: Imprenta Nacional.

Schiaffino, R. (1928). Cuerpo Médico Escolar. En E. Acevedo (1928), Memoria de Instrucción Primaria correspondiente al año 1927. Parte segunda (pp. 267-278). Montevideo, Uruguay: Imprenta Nacional.

Schiaffino, R. (1928). El Cuerpo Médico Escolar. En E. Acevedo (1929), Memoria de Instrucción Primaria correspondiente a 1928 (pp. 119-137). Montevideo, Uruguay: Imprenta Nacional.

Varela, J. P. (1964). La educación del pueblo. Montevideo, Uruguay: Ministerio de Instrucción Pública y Previsión Social.

Vidal de Olivet, M. (1918). Economía doméstica. Anales de Instrucción Primaria, Año XV-XVI, Tomo XV, nos. 112, Julio de 1917 a junio de 1918. Montevideo, Uruguay: El Siglo Ilustrado, pp. 496-522.

\section{Contribución autoral}

a) Concepción y diseño del trabajo; b) Adquisición de datos; c) Análisis e interpretación de datos; d) Redacción del manuscrito; e) revisión crítica del manuscrito.

F. S. ha contribuido en a, b, c, d, e.

Editora científica responsable: Mag. Florencia de León 